\title{
The genomic Echinococcus microsatellite EmsB sequences: from a molecular marker to the epidemiological tool
}

\author{
J. KNAPP ${ }^{1,2}$, J. M. BART ${ }^{1}$, S. MAILLARD ${ }^{1,2}$, B. GOTTSTEIN ${ }^{2 *}$ and R. PIARROUX ${ }^{1,3}$ \\ ${ }^{1}$ Laboratory of Chrono-Environment, Department of Parasitology, UMR CNRS 6249, usc INRA, \\ University of Franche-Comté, 19 rue Ambroise Paré, 25030 Besançon, France \\ ${ }^{2}$ Institute of Parasitology, Vetsuisse Faculty, University of Bern, Längass-Strasse 122, 3012 Bern, Switzerland \\ ${ }^{3}$ Department of Parasitology and Mycology, Hôpital la Timone, 13005 Marseille, France
}

(Received 7 August 2009; revised 9 October 2009; accepted 10 October 2009; first published online 22 December 2009)

\section{S U M MARY}

In the field of molecular and epidemiological parasitology, characterization of fast evolving genetic markers appears as an important challenge to consider the diversity and genetic structure of parasites. The study of respective populations can help us to understand their adaptive strategies to survive and perpetuate the species within different host populations, all trying to resist infection. In the past, the relative monomorphic features of Echinococcus multilocularis, the causative agent of alveolar echinococcosis and a severe human parasitic disease, did not stimulate studies dealing with the genetic variability of Echinococcus species or respective populations. A recently developed, characterized and validated original multilocus microsatellite, named EmsB, tandemly repeated in the genome, offered an additional opportunity for this line of investigation. We have compiled in this review new insights brought by this molecular tracker on the transmission activity of Echinococcus among different hosts and at different geographical scales.

Key words: Echinococcus multilocularis, highly polymorphic molecular marker, microsatellite EmsB, population dynamic activity, transmission pattern, eco-epidemiology.

\section{INTRODUCTION}

One area of major advancement in helminthology has been in understanding genetic and molecular parameters of parasite populations. To a large extent, this progress follows significant developments of molecular markers that are widely used mainly in two fields. Firstly, the study of the genetic evolution of a species and the examination of its taxonomic relationships with other organisms have both been tackled. Secondly, ecology was contracted to identify an organism, investigate population genetics or to consider its dynamic activity within a given environment (Monis et al. 2002). Due to the important veterinary and medical significance of cestodes, efficient molecular tools have been explored for many species to improve detection, prevention and management of zoonotic transmission in particular (Yang et al. 2005; Smith, 2009). Echinococcus multilocularis is the causative agent of the human alveolar echinococcosis (AE), and is one of the most threatening parasitic zoonoses of the Northern hemisphere. Its transmission pattern involves different mammalian hosts. Foxes (Vulpes vulpes) and dogs (Canis lupus familiaris) act as the main definitive

* Corresponding author: Institute of Parasitology, Vetsuisse Faculty, University of Bern, Längass-Strasse 122, 3012 Bern, Switzerland. Tel: +41(31)6312476. Fax: +41(31)6312622. E-mail: bruno.gottstein@ipa.unibe.ch hosts (DH). A wide range of small-mammals, including mainly rodents such as Arvicola spp. and Microtus spp. and Lagomorpha of the genus Ochotona act as intermediate hosts (IH) (Vuitton et al. 2003). Cystic echinococcosis (CE) is a widespread and severe zoonosis caused by infection with the larval stage of Echinococcus granulosus sensu lato (E. granulosus s.l.). As with $\mathrm{AE}$, humans acquire $\mathrm{CE}$ infections by ingesting Echinococcus eggs via contaminated food or water, through close physical contacts with DH, or directly with faeces of these hosts. Its transmission pattern also involves different mammalian hosts, mainly canids as $\mathrm{DH}$ and a large variety of livestock animals as IH. Based on mitochondrial DNA studies, the classification within the E. granulosus s.l. paraphyletic taxon has undergone, and continues to undergo, important changes. E. granulosus s.l. is composed of heterogeneous groups of variants, defined as 'strains' (G1 to G10) (Bowles et al. 1992; Bowles and McManus, 1993; Scott et al. 1997 ; Lavikainen et al. 2003). However, these strains are now reorganized within distinct species (Thompson and McManus, 2002; Lavikainen et al. 2003; Nakao et al. 2007). E. granulosus sensu stricto ( $E$. granulosus s.s.) encompasses the G1, G2 and G3 strains, E. equinus corresponds to the G4 strain, and E. ortleppi comprises the G5 strain. The G6, G7, G8, G9 and G10 strains have also been classified under a well-supported monophyletic species, E. canadensis (Nakao et al. 2007). Moreover, 


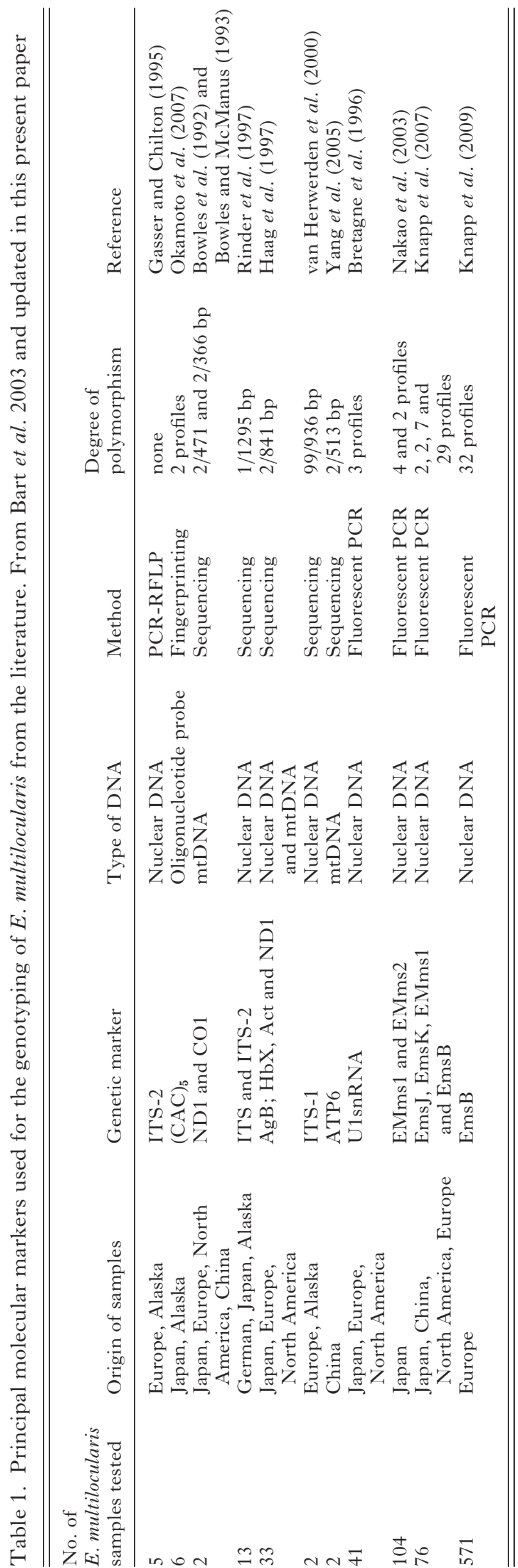

this revised classification seems to be more in accordance with nuclear studies, which emphasizes smaller differences than observed in mtDNA studies among the E. granulosus s.l. stains (Saarma et al. 2009).

Molecular tools are required to better understand taxonomic relationships between Echinococcus species and infra-specific taxa, as well as to precise molecular tracking of Echinococcus isolates collected from humans and animals based upon geographically or biologically relevant markers. However, so far, molecular studies have exhibited very little genetic variation within E. multilocularis. Notwithstanding the methods used, most molecular markers lacked of discriminatory power. In order to increase the level of resolution and sensitivity of the tools, we set up an original molecular target represented by a highly tandemly repeated microsatellite, called EmsB. In contrast to classical microsatellite targets, PCR amplification of EmsB provided a multi-peak profile, characterized by tandemly repeated microsatellite sequences. This aspect confers to EmsB a higher discriminatory power than all of the currently available molecular tools (see Table 1).

The present paper reviews the different steps which have led to the discovery, characterization and application of this original molecular tool for the evaluation of genetic polymorphism in E. multilocularis and E. granulosus s.l. Its application in several molecular epidemiological studies has suggested new hypotheses on the dispersion pattern of E. multilocularis in endemic foci, especially in the European focus, controversially described as a centrally (Alpine arch area) historical endemic area, and in its peripheries as newly endemic zones (Eckert et al. 2000). It also provided new insights in the molecular epidemiology of E. granulosus s.l. and in the demonstration of cross-fertilization between the new defined species of E. granulosus s.s. and E. ortleppi.

THE NEED FOR HIGHLY POLYMORPHIC MARKERS WITHIN THE GENUS ECHINOCOCCUS

Despite substantial efforts to develop and apply valuable genetic tools for $E$. multilocularis genotyping (Table 1) (Bowles et al. 1992; Bowles and McManus, 1993 ; Gasser and Chilton, 1995 ; Bretagne et al. 1996; Haag et al. 1997; Rinder et al. 1997; van Herwerden et al. 2000; Nakao et al. 2003; Yang et al. 2005 ; Knapp et al. 2007 ; Okamoto et al. 2007), a high discriminatory power was not really achieved except when using microsatellite targets. Overall, an important homology was described within the species E. multilocularis, which could probably be explained first by an almost unique reproduction pattern at the adult stage by homogamy (Haag et al. 1997, 1998) and secondly by the recent report of E. multilocularis and the evolutionary history of the genus (Bowles 


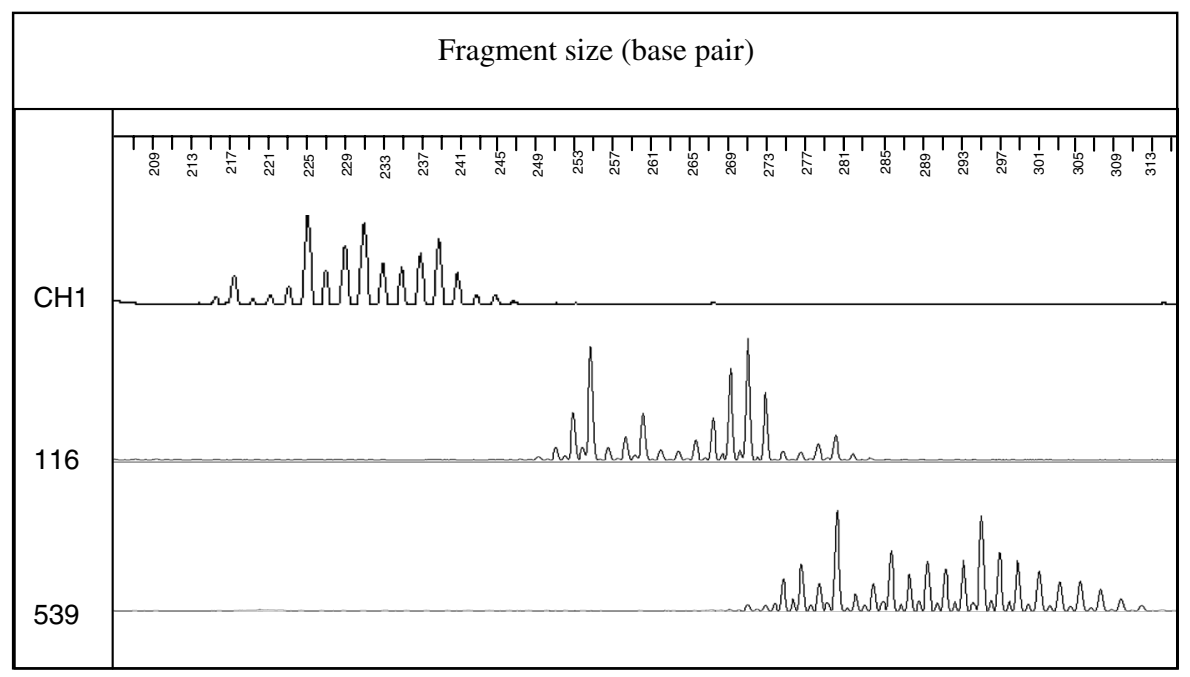

Fig. 1. Fragment size analyses of the EmsB target, realized on DNA samples of E. multilocularis from Switzerland (CH1), E. canadensis (116), from Mauritania and E. granulosus sensu stricto (539), from Algeria, with the ABI Prism 3100 sequencer.

and McManus, 1993). It was necessary to search for rapidly evolving DNA fragments. The first study devoted to E. multilocularis genotyping using microsatellite considered the U1 snRNA multi-gene, tandemly repeated in DNA (around 50 copies in the Eukaryote genome), separated by a pentameric microsatellite (Bretagne et al. 1991). Analysing samples from North America, Japan and Europe, three profiles were described (a profile was described as a series of peaks on the electropherogram). A regional polymorphism was described and similar profiles were found between North America and Japan, suggesting a putative common origin of E. multilocularis (Bretagne et al. 1996). The second study focused on 2 single-locus microsatellites, EMms1 (GenBank Access No. AB100031, composed of an array of different triplets) and EMms2 (AB100032 composed of one motif $\left.(\mathrm{CAC})_{n}\right)$ (Nakao et al. 2003). These two microsatellites were used with worms originating from the endemic island of Hokkaido, Japan. Four genotypes were described for EMms1 and two for EMms2. Mixed infections in foxes were also found (worm burdens contained parasites of different genotypes). Unfortunately and in spite of a high level of genetic diversity, it was not possible to link the E. multilocularis allelic frequency to geographical position of foxes, due to the insular origin of samples. In comparison with $E$. multilocularis, E. granulosus s.l. is less monomorphic and sequencing of some mitochondrial and nuclear DNA targets revealed important genetic diversity, leading to the definition of the above mentioned strains and new species. However, mitochondrial and nuclear markers were not sufficiently polymorphic to identify genetic variations that reflect regional or focal population characteristics. To date, only four singlelocus microsatellites have been used to assess E. granulosus s.l. genetic diversity: U1snRNA,
EgmSca1, EgmSca2 and EgmSga1. The U1snRNA gene provided 11 distinct profiles among species: 8 for E. granulosus s.s. (G1/G2), 2 for E. ortleppi (G5) and 1 for E.canadensis (G6) (Roratto et al. 2006), but no spatial correlation was observed. Among the three microsatellites described in Bartholomei-Santos and co-workers (Bartholomei-Santos et al. 2003), only EgmSca1 provided results indicating a weak spatial correlation in 73 Brazilian and Argentinean samples.

ISOLATION AND CHARACTERISATION OF EMSB

The search for highly polymorphic markers was continued and targeted on new microsatellite sequences that would meet the expected level of discriminatory sensitivity (Bart et al. 2006a). After screening an E. granulosus DNA library, 17 microsatellites were identified. This species was used because, at that time, only protoscoleces obtained from hydatid cysts yielded high quantities of pure material without host DNA contamination. From a first inter-species screening, 3 microsatellite targets were found to be polymorphic: EmsJ (GenBank ref. No. AY680845) presenting a repeated motif $(\mathrm{CT})_{\mathrm{n}}$, EmsK (GenBank ref. No. AY680857) with a repeated motif $(\mathrm{CA})_{\mathrm{n}}$ and $\mathrm{EmsB}$ with two repeated motifs $(\mathrm{CA})_{\mathrm{n}}(\mathrm{GA})_{\mathrm{m}}$ ( $\mathrm{n}$ and $\mathrm{m}$, number of repetitions). In comparison to the other microsatellites, EmsB presented a peculiar electropherogram profile, with a notable difference among Echinococcus species and isolates tested (Fig. 1). Based on this finding it was decided to unravel the genetic structure of EmsB in order to explain its non-conventional profile.

The strategy to elucidate the nature of the multipeak electropherogram was to isolate by cloning the EmsB PCR products. The screening of 110 clones and analyses of the fragment sizes of each inserted 


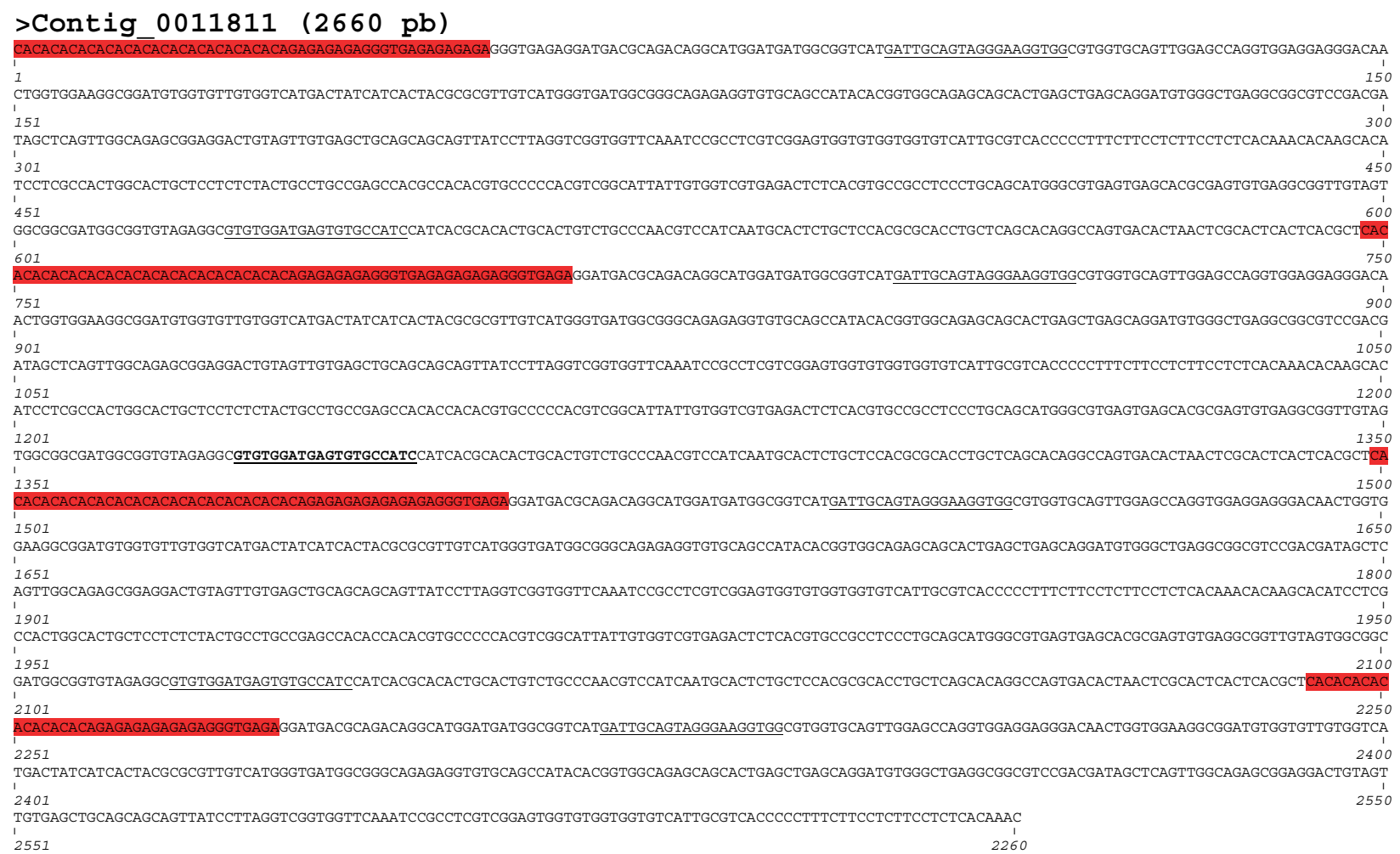
2551

Fig. 2. Sequence no. 11811 of 2660 bp published in the E. multilocularis Genome Project by the Sanger Institute presenting 4 microsatellite EmsB sequences found tandemly repeated. Repeated motifs are highlighted in red and forward/reverse primers are in green. For the first microsatellite (position 1 to $115 \mathrm{bp}$ ), only the reverse primer was found.

PCR product allowed us to observe single peaks, discarding a possible in vitro slippage of the polymerase during the PCR. The reconstitution of an EmsB profile by addition of the isolated peaks yielded a profile similar to the original one. The sequencing of isolated fragments showed a slight homoplasy phenomenon among fragments of the same size which could be interpreted as an inner polymorphism between the different loci. This phenomenon leads to a loss of information, but the highest level of data provided by a multi-locus marker could easily compensate for this problem (Estoup et al. 2002). The complex electropherogram profile of the EmsB target could be due to its presence in multi-copies in the Echinococcus DNA and not to an artefact. This hypothesis was confirmed by quantitative PCR and the number of copies of EmsB was evaluated at $10^{4}$ copies in the Echinococcus genome (Fig. 2).

To assess the distribution of this microsatellite in the genome, PCRs with large elongation steps and different primer sets were performed. Several bands, regularly spaced were amplified, that suggested a tandem repetition of the target. The presence of EmsJ, EmsK and EmsB was searched in the recently published genome sequence for $E$. multilocularis from the Sanger Institute in the framework of the Echinococcus Genome project (http://www.sanger.
ac.uk/Projects/Echinococcus). EmsJ and EmsK were found in a single copy in the $E$. multilocularis data base, in the contig sequences No. 4805 and 18680 respectively. EmsB was found among several short contig sequences and for some of them tandemly repeated (e.g. contig No. 11811 where EmsB A/C was found 4 times (Fig. 2) and contig No. 19011, 2 times (data not shown)).

The EmsB PCR products could be characterized by fragment size analyses. Electropherograms revealed a series of peaks, which were called EmsB profiles. Two parameters on the electropherogram were retained to characterize each EmsB profile: the size (in base pairs) of the amplified fragments and the number of targets of the same size were revealed by the intensity of the peaks. The Euclidian distances between two computed profiles could be calculated in order to assess the similarity/dissimilarity among a sample collection. The hierarchical clustering analysis using an unweighted-pair group method and average linkage (UPGMA) (Legendre and Legendre, 1998) yielded dendrograms graphically representing the relationships among samples; the robustness was tested by bootstrap resampling.

To complete the characterization of EmsB, stability in time of the microsatellite, reproducibility, repeatability, sensitivity and specificity of its amplification by PCR were all assessed. For stability, three 
isolates from the E. multilocularis strain F AUB-2, maintained one year in vivo in Meriones unguiculatus were analysed at three different times. The three profiles were highly similar and used to define a genetic distance threshold that discriminates the EmsB profiles. This genetic distance was further used for all the epidemiological surveys performed with EmsB. For reproducibility and repeatability, different DNA polymerases and different conditions of amplification were tested. Reproducibility and repeatability were also tested in different laboratories and by different operators and have provided valid results (Bart et al. 2006b). The sensitivity was described on agarose gel with $1 \mathrm{fg}$ of DNA. The specificity was tested on host DNA (human, and mouse (Bart et al. 2006 b), foxes (Knapp, 2008)) and on a panel of helminths (Casulli et al. 2009). Only isolates from the genus Echinococcus yielded appropriate amplifications.

\section{FROM GENOME TO FIELD STUDIES}

The usefulness of EmsB to study the genetic diversity of E. multilocularis and E. granulosus with a multi-scale approach was assessed. Three main questions needed to be addressed: (1) Is the polymorphism of the marker sufficient to obtain a molecular signature to recognised 'strain' circulating among hosts, without over-discrimination and independent to hosts? (2) What are the geographical limits of its genetic discrimination? (3) Is the marker useful to study the spatial structure and the dynamic activity of the parasite at different geographical ranges/levels?

The power of the EmsB marker in the search for E. multilocularis genetic polymorphism was realized with a global collection composed of 76 isolates from humans, monkeys, rodents and foxes, originating from North America (Saint Lawrence island in Alaska and Canada), China (Tibetan plateau), Japan (Hokkaido island) and Europe (Switzerland, France, Germany, Austria, Poland, Czech Republic, Slovakia and the Netherlands) (Knapp et al. 2007). Finally with the EmsB marker, 29 genotypes were described (Fig. 3) and regional groups (European, North American, Chinese and Japanese groups) were graphically defined, including 23 clusters among the European samples. Same EmsB profiles were found shared as well among $\mathrm{DH}$ (foxes) as among $\mathrm{IH}$ (humans and monkeys). This finding underlined the independence of EmsB profiles for hosts. To the best of our knowledge, this paper related the highest genetic diversity described for E. multilocularis on a worldwide panel. These findings lead us to believe that EmsB could be employed to retrieve the genetic diversity of individual worms and study the distribution of its genetic variability at different spatial ranges, independent of hosts and investigate the transmission activity of the parasite.
FIELD STUDIES

Further investigations targeted the E. multilocularis genetic diversity with a multi-geographical-scale approach. In order to assess the genetic discrimination threshold of the marker EmsB and its power to describe circulation of the parasite among different host populations, three geographical scales were investigated: (1) at a regional scale approach, taking into consideration the interaction of several fox populations; (2) at two local scale approaches (territory of a fox population), in two different topographical situations, first in an undulating area in the French Ardennes (North of France) and in a mountain area in the Val Pusteria (North of Italy); and finally (3) at a micro-local scale, by investigating parasites isolated from close related rodent hosts, collected in a very limited field (around $100 \mathrm{~m}^{2}$ ) in Switzerland and independently in Alaska, to assess the limits of the EmsB discrimination power for closely related samples.

THE ECHINORISK PROJECT: EmsB STUDY TO ADDRESS THE GENETIC DIVERSITY OF E. MULTILOCULARIS AT THE CONTINENTAL SCALE IN EUROPE

In Europe, E. multilocularis is historically described as predominantly occurring on the North side of the Alpine arch (East of France, South Germany, Austria and Switzerland), essentially because most of human records are described in this area (Eckert et al. 2000 ; Kern et al. 2003). In order to study the pattern of occurrence E. multilocularis in Europe, a collection of 571 adult worms from 123 geo-referenced foxes (Fig. 4) were sampled in 9 European regions, including the historical core (Switzerland, North Austria, Germany (Swabian Jura and Bavaria) and West Czech Republic) and 4 areas considered as newly endemic regions (North of France, Central Slovakia, Tatra mountains and North Poland) (Knapp et al. 2009). This study described a total of 32 EmsB profiles. Among them, six profiles represented $69 \%$ of the whole sample collection and 5 of them were widely described in the European territory surveyed. The sampling effort was tested by a rarefaction analysis (Colwell, 2005) showed that the genetic richness was not reached in almost all studied sub-regions (Fig. 5). However, the genetic diversity was found to be higher in the historical endemic area compared to peripheral zones. Moreover, in each peripheral zone, one profile was found to be dominant. This profile was also found in the historically endemic area but was harboured by only a few specimens. This finding prompted the occurrence of a founder event, where a numerically low represented species or a genotype exported to a hitherto free territory could be found dominant after colonization, followed by its spreading (Templeton, 
Fragment size (base pair)

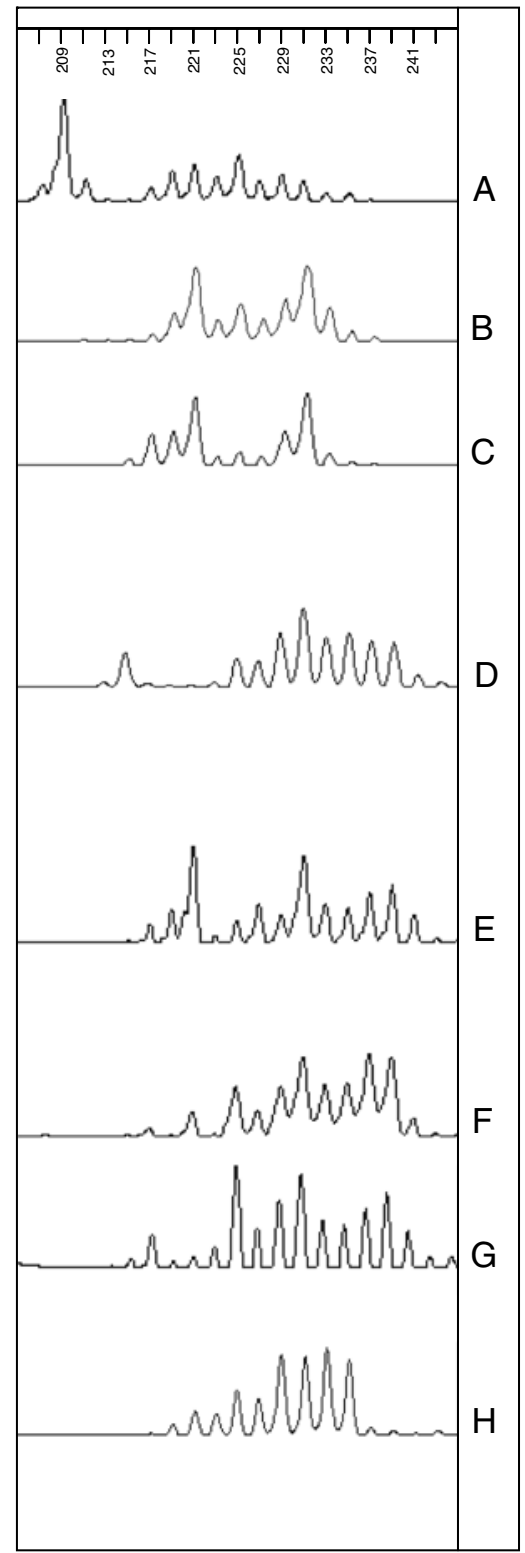

a

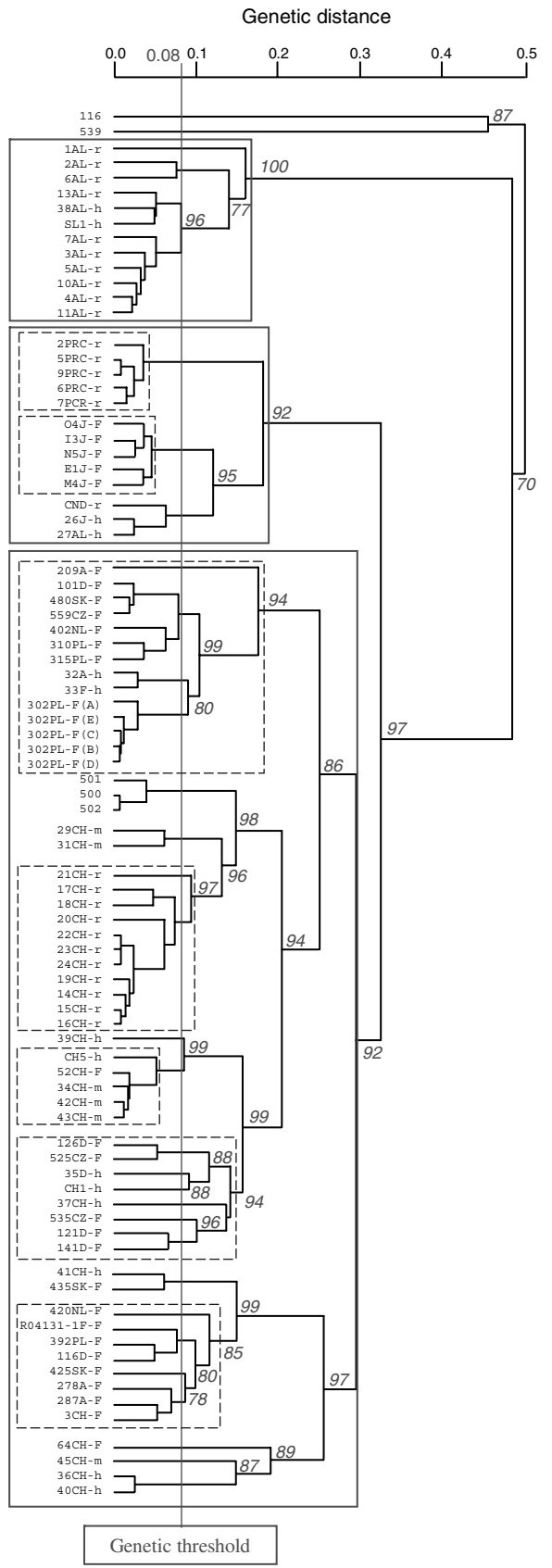

b

Fig. 3. E. multilocularis genetic classification according to EmsB results. On the left side (a), example of electropherograms of EmsB loci (209 bp to $241 \mathrm{bp}$ ), using the automatic sequencer ABI Prism $3100^{\mathrm{TM}}$. On the right side (b), dendrogram based on EmsB genotypic data, constructed by hierarchical clustering analysis (Euclidian distance, average link clustering method), with pvclust, under R Project. The approximately unbiased $P$ values (numbers on nodes, in percent) were calculated with a multiscale bootstrap $(B=1000)$. The three solid-line boxes show St. Lawrence Island's samples (upper box), the Asian-North American samples (middle box) and the European samples (lower box). The electropherograms correspond in the genetic tree to (A) the St. Lawrence Island profile and the dotted-line boxes refer to (B) the Chinese rodent profile, (C) the Japanese fox profile, and (D) through (H) the main European profiles. Samples 500, 501 and 502 represent a single isolate maintained in vivo by several passages in Meriones unguiculatus. Sample 116 originating from a Mauritanian camel and sample 539 originating from an Algerian sheep were E. granulosus samples and were included as an out-group control (redrawn from (Knapp et al. 2007)).

2008). Distinctive features of local parasite cycles, e.g. involvement of peculiar intermediate hosts, landscapes or effect of human behaviours (agriculture, deforestation, occupation of fox territory, etc.) could also have an important role in the parasite genotype distribution into the environment 


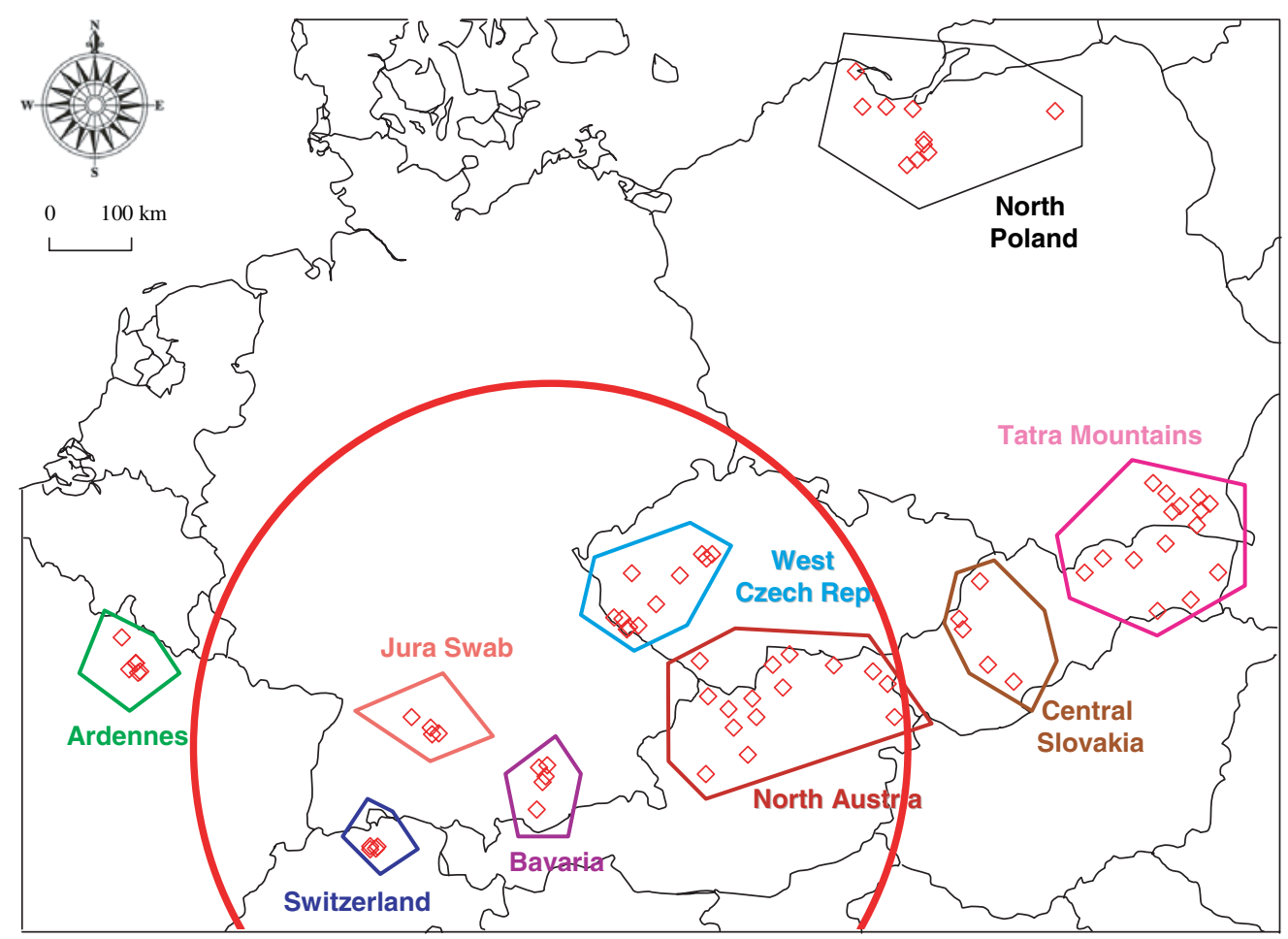

Fig. 4. Spatial distribution of various samples of E. multilocularis collected from infected foxes in Europe, and clustered according to geographical peculiarities. Red lozenges represent the position of fox; polygons define a geographically restricted sub-region. The red circle area indicates the historically documented E. multilocularis central endemic focus in Europe, where most humans cases have been recorded in the past five decades (redrawn from Knapp et al. 2009).

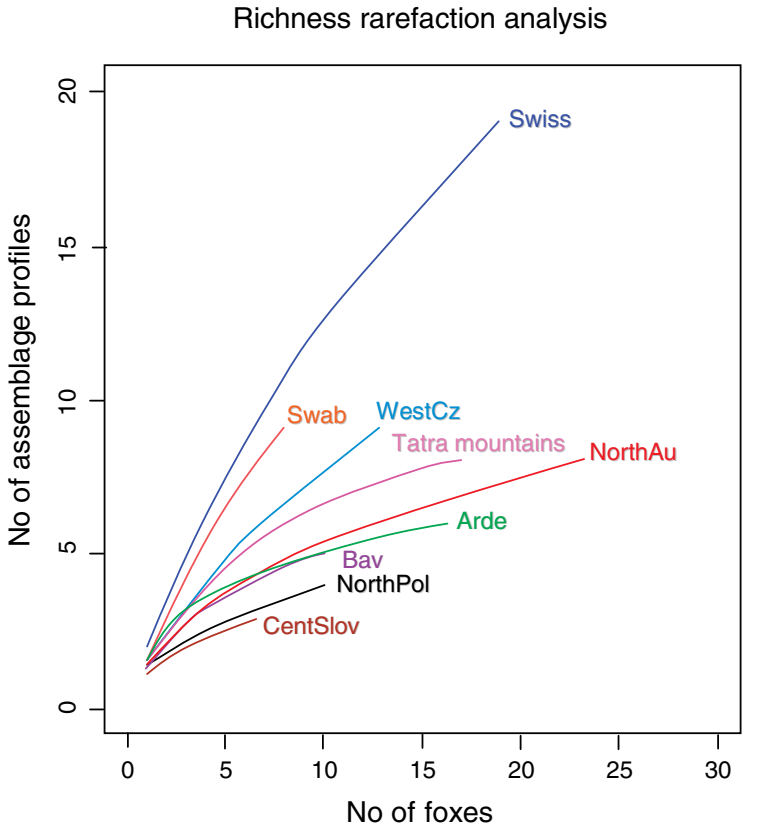

(a)

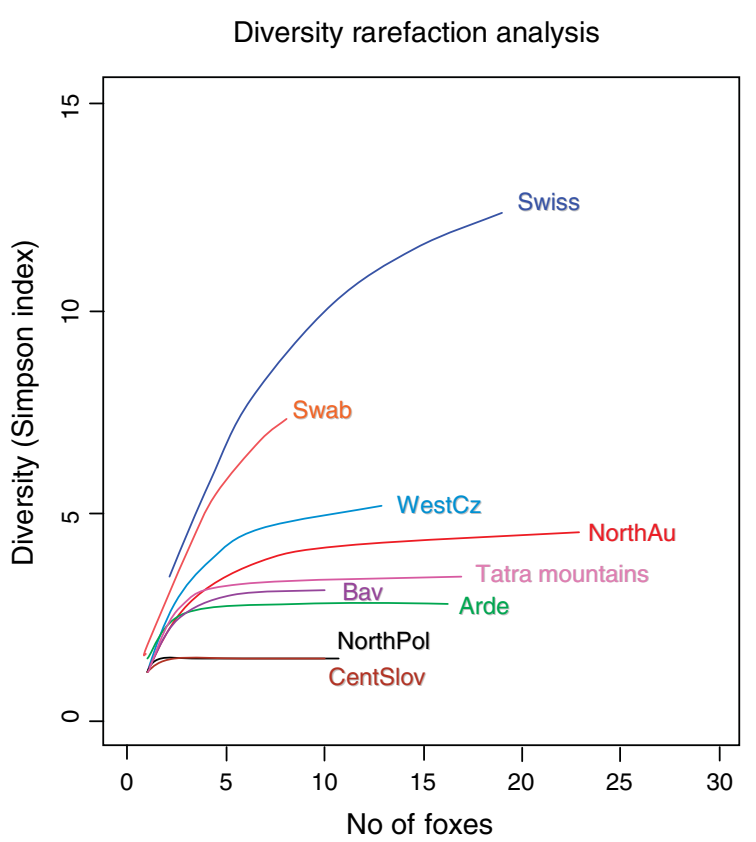

(b)

Fig. 5. Genetic richness (a) and diversity (Inverse Simpson index, b) rarefaction curves for each sub-region (fox as a sampling unit). Swiss: Switzerland, canton of Zurich; NorthAu: North Austria; Swab: Swabian Jura; Bav: Bavaria; Arde: Ardennes; WestCz: West Czech Republic; NorthPol: North Poland; EastSlovPol: East Slovakia and South Poland; CentSlov: Central Slovakia (redrawn from Knapp et al. 2009). 
as it was suspected in the French Ardennes region (Knapp et al. 2008). Overall, findings suggest that the dispersion pattern of E. multilocularis in the endemic European focus could be attributed to a mainlandisland spreading and dominant genotypes in peripheral regions of the historical area explained by founder events. Regarding the slow dispersion of the parasite in Europe (Takumi et al. 2008), this phenomenon had certainly required longer time than previously reported.

LOCAL SCALE: E. MULTILOCULARIS

GENETIC DIVERSITY TRANSMITTED AMONG

FOX COMMUNITIES

Several independent studies have documented the value of EmsB to assess the genetic diversities and peculiarities of local E. multilocularis populations within the definitive host. One study was carried out in a mountain area, the Val Pusteria in the North Italy (Casulli et al. 2009). When compared to 32 different EmsB profiles described for Central Europe (Knapp et al. 2009), this North Italian region presented only 4 endemic profiles, which suggested the occurrence of a local parasite life cycle in this region independent of South Austria (Knapp et al. 2007; Casulli et al. 2009). The presence of the parasite in the South Alpine region might be explained by the spread of E. multilocularis from the historical core described in the Alpine Arch. As observed in Europe, a founder event probably due to fox dispersion through the Alpine mountain could have permitted the establishment of the E. multilocularis genotypes found in this Italian area, after their proliferation in local foxes (Templeton, 2008). But as these Italian genotypes were not found in neighbouring Austria (Knapp et al. 2007, 2009) one might suspect other origins of the parasites, e.g. from other areas in Italy. Moreover, one cannot exclude an historic presence of E. multilocularis in the Southern part of Europe, away from the Alps. The recent findings suggest intensive drift of E. multilocularis in Europe for a longer time than was previously thought. Moreover the reports of five cases in Italy more than 100 years ago (Posselt, 1900) confirmed the ancient presence of the parasite in the Southern Alps. But if few exchanges through the Alps occur, the parasite genotype frequency could decrease by a bottleneck phenomenon, due to the mountainous area (Ricklefs and Miller, 2000).

Furthermore, a locally restricted genetic diversity of E. multilocularis was highlighted in a newly recognised endemic region in the Northern part of France (the French Ardennes region). The prevalence of E. multilocularis in this region infected $53 \%$ of foxes (Guislain et al. 2008), and considered as highly endemic. From 140 individual adult worms, collected from 25 foxes, six EmsB profiles were described (Knapp et al. 2008). Genotypically mixed infections were confirmed in foxes, as previously shown in the endemic island of Hokkaido by using the single-locus microsatellite EMms1 (Nakao et al. 2003), and EmsB in Europe. A single fox can harbour between 1 to at least 4 different genotypes (Nakao et al. 2003; Knapp et al. 2008; Casulli et al. 2009), which could depend on the intensity of predation of contaminated rodents and also on the size of the territory investigated by the fox. The simultaneous presence of genotypically different sub-populations of $E$. multilocularis in fox intestines suggests the possible occurrence of mating events between worms from different populations and yielding of hybrid eggs. Nevertheless the phenomenon was detected only very rarely in collected foxes.

CIRCULATION OF E. MULTILOCULARIS

AT A MICRO-LOCAL SCALE

Among our previous sample collection (Knapp et al. 2007), two panels of naturally infected rodents were collected from fields a few hectares in area in both Switzerland and Alaska were available. The genetic diversity among these related samples was the lowest, and due to these limited genetic differences between samples, these formers were considered to be the most biologically related isolates. One might suspect a common infection of rodents by a unique fox or their contamination after the transmission of a single E. multilocularis strain. These findings inform us of the limits of the genetic discrimination of the EmsB marker and we strongly believe that the EmsB genotyping avoided an over-discrimination even for geographically close related samples (Knapp et al. 2007).

THE MICROSATELLITE EmsB: A NEW TOOL TO INVESTIGATE THE GENETIC DIVERSITY OF ECHINOCOCCUS GRANULOSUS SENSULATO

Recently, EmsB was used to characterize 125 samples of E. granulosus s.l. originating from various different host species (sheep, cattle, dromedaries, dogs and human patients) collected in six different countries (Algeria, Mauritania, Romania, Serbia, Brazil and People's Republic of China) (Maillard et al. 2009). The conventional mitochondrial cox1 and nad1 markers permitted identification of the genotypes G1, G3, G5, G6 and G7, which were clustered into three groups corresponding to the species E. granulosus s.s., E. ortleppi and E. canadensis. The polymorphism exhibited by these markers did not reach the level necessary for the identification of genetic variants within restricted geographical areas. Based on the same samples, EmsB provided a higher genetic discrimination and identified a continental and an intercontinental circulation of the G1 genotypes, the common sheep strain. The trading of the hosts (local and worldwide) 
and the local migration of (stray) dogs may explain these two types of circulation. Furthermore, the microsatellite EmsB may be potentially useful for taxonomic consideration. The description of mixed G1/G5 profile with EmsB indicated the occurrence of genetic exchanges between distinct $E$. granulosus s.s. and E. ortleppi populations circulating in Brazil. This result suggests the absence of a strong reproductive barrier between the two species. However these findings encourage further genetic investigations. To summarize, EmsB may be useful for the assessment of the genetic polymorphism of E. granulosus s.l. as well as its taxonomy and details of its spatial distribution. Knapp and co-workers (2007, 2008) had already shown earlier that this microsatellite enriched a powerful panel of markers to track temporal and spatial transmission of $E$. multilocularis. In this work, the microsatellite EmsB also exhibited an interesting potential for E. granulosus $s . l$. because it could be useful for the elaboration of a detailed distribution map of genetic variants and in the determination and tracking of the infection source of $\mathrm{CE}$.

\section{CONCLUSION}

In population genetics, with special reference to ecoepidemiology, the development of highly polymorphic tools is necessary to trace infectious organisms in the environment. The parasites E. multilocularis and E. granulosus s.l. appeared as interesting models, because they need two mammalian hosts to perpetuate, with a large range of intermediate hosts. Echinococcus spp. are transported widely by carnivores, the predators of its intermediate hosts, but present a relatively low intraspecific genetic diversity in DNA coding regions. Silent mutations could be analysed to define the evolution of the parasite. EmsB is a novel genetic multi-locus tool and, due to its presence in multi-copies within the Echinococcus genome, offers appropriate methodological sensitivity to trace the genetic variability of $E$. $m u l$ tilocularis at a hitherto unattainable level. Nevertheless, we have to keep in mind that such research tools provide data which must finally be integrated into a global approach. Numerous parameters have to be taken into account in this analysis, such as geographical data, eco-epidemiology and also human and veterinary health monitoring strategies. These multi-factor studies enabled us to gain a better understanding of the dispersion of an organism in the environment, and the threat it represents to humans. From an eco-epidemiological perspective, one of the crucial future steps to progress molecular-genetic research will be to include human $\mathrm{AE}$ and $\mathrm{CE}$ patients in this type of topic. The genotyping of parasites transmitted to humans could putatively be used as a complementary tool in health control programmes.

\section{ACKNOWLEDGMENTS}

We are very grateful to the different laboratories and institutes where this work was undertaken and for technical help and support: to Prof. Christiane Mougin and Dr Laurent Bermont from the Laboratory of Biochemestry and Cellular Biology, University Hospital, Besançon, France, to Dr Marie-Louise Glowatzki from the Department of Clinical Research, Vetsuisse faculty, Berne, Switzerland. We also thank Karen Haag for constructive comments on our works and Prof. Ito from the Asahikawa Medical College for his support.

\section{REFERENCES}

Bart, J. M., Abdukader, M., Zhang, Y. L., Lin, R. Y., Wang, Y. H., Nakao, M., Ito, A., Craig, P. S., Piarroux, R., Vuitton, D. A. and Wen, H. (2006a). Genotyping of human cystic echinococcosis in Xinjiang, PR China. Parasitology 133, 571-579.

Bart, J. M., Knapp, J., Gottstein, B., El-Garch, F., Giraudoux, P., Glowatzki, M. L., Berthoud, H., Maillard, S. and Piarroux, R. (2006b). EmsB, a tandem repeated multi-loci microsatellite, new tool to investigate the genetic diversity of Echinococcus multilocularis. Infection, Genetics and Evolution 6, 390-400.

Bartholomei-Santos, M. L., Heinzelmann, L. S., Oliveira, R. P., Chemale, G., Gutierrez, A. M., Kamenetzky, L., Haag, K. L. and Zaha, A. (2003). Isolation and characterization of microsatellites from the tapeworm Echinococcus granulosus. Parasitology 126, 599-605.

Bowles, J., Blair, D. and McManus, D. P. (1992). Genetic variants within the genus Echinococcus identified by mitochondrial DNA sequencing. Molecular and Biochemical Parasitology 54, 165-173.

Bowles, J. and McManus, D. P. (1993). Molecular variation in Echinococcus. Acta Tropica 53, 291-305.

Bretagne, S., Assouline, B., Vidaud, D., Houin, R. and Vidaud, M. (1996). Echinococcus multilocularis: microsatellite polymorphism in U1 snRNA genes. Experimental Parasitology 82, 324-328.

Bretagne, S., Robert, B., Vidaud, D., Goossens, M. and Houin, R. (1991). Structure of the Echinococcus multilocularis U1 snRNA gene repeat. Molecular and Biochemical Parasitology 46, 285-292.

Casulli, A., Bart, J. M., Knapp, J., La Rosa, G., Dusher, G., Gottstein, B., Di Cerbo, A., Manfredi, M. T., Genchi, C., Piarroux, R. and Pozio, E. (2009). Multi-locus microsatellite analysis supports the hypothesis of an autochthonous focus of Echinococcus multilocularis in northern Italy. International Fournal for Parasitology 39, 837-842.

Colwell, R. K. (2005). EstimateS : Statistical estimation of species richness and shared species from samples. Version 7.5. User's Guide and application published at: http://purl.oclc.org/estimates.

Eckert, J., Conraths, F. J. and Tackmann, K. (2000). Echinococcosis: an emerging or re-emerging zoonosis? International Fournal for Parasitology 30, 1283-1294.

Estoup, A., Jarne, P. and Cornuet, J.-M. (2002). Homoplasy and mutation model at microsatellite loci 
and their consequences for population genetic analysis. Molecular Ecology 11, 1591-1604.

Gasser, R. B. and Chilton, N. B. (1995). Characterisation of taeniid cestode species by PCR-RFLP of ITS2 ribosomal DNA. Acta Tropica 59, 31-40.

Guislain, M. H., Raoul, F., Giraudoux, P., Terrier, M. E., Froment, G., Ferte, H. and Poulle, M. L. (2008). Ecological and biological factors involved in the transmission of Echinococcus multilocularis in the French Ardennes. Fournal of Helminthology 82, $143-151$

Haag, K. L., Araujo, A. M., Gottstein, B. and Zaha, A. (1998). Selection, recombination and history in a parasitic flatworm (Echinococcus) inferred from nucleotide sequences. Memorias do Instituto Oswaldo Cruz 93, 695-702.

Haag, K. L., Zaha, A., Araujo, A. M. and Gottstein, B. (1997). Reduced genetic variability within coding and non-coding regions of the Echinococcus multilocularis genome. Parasitology 115, 521-529.

Kern, P., Bardonnet, K., Renner, E., Auer, H., Pawlowski, Z., Ammann, R. W. and Vuitton, D. A. (2003). European echinococcosis registry: human alveolar echinococcosis, Europe, 1982-2000. Emerging Infectious Diseases 9, 343-349.

Knapp, J. (2008). Caractérisation et validation du marqueur microsatellite multilocus répété en tandem EmsB pour la recherche de polymorphisme génétique chez Echinococcus multilocularis: Application à l'étude de la transmission du parasite en Europe. PhD Thesis, University of Franche-Comté.

Knapp, J., Bart, J. M., Giraudoux, P., Glowatzki, M. L., Breyer, I., Raoul, F., Deplazes, P., Duscher, G., Martinek, K., Dubinsky, P., Guislain, M. H., Cliquet, F., Romig, T., Malczewski, A., Gottstein, B. and Piarroux, R. (2009). Genetic diversity of the cestode Echinococcus multilocularis in red foxes at a continental scale in Europe. PLoS Neglected Tropical Diseases 3, e452.

Knapp, J., Bart, J. M., Glowatzki, M. L., Ito, A., Gerard, S., Maillard, S., Piarroux, R. and Gottstein, B. (2007). Assessment of microsatellite polymorphism for improving the spatial distribution tracking of Echinococcus multilocularis. Fournal of Clinical Microbiology 45, 2943-2950.

Knapp, J., Guislain, M. H., Bart, J. M., Raoul, F., Gottstein, B., Giraudoux, P. and Piarroux, R. (2008). Genetic diversity of Echinococcus multilocularis on a local scale. Infection, Genetics and Evolution 8, 367-373.

Lavikainen, A., Lehtinen, M. J., Meri, T., Hirvela-Koski, V. and Meri, S. (2003). Molecular genetic characterization of the Fennoscandian cervid strain, a new genotypic group (G10) of Echinococcus granulosus. Parasitology 127, 207-215.

Legendre, P. and Legendre, L. (1998). In Numerical Ecology, 2nd English edition. Elsevier Science BV, Amsterdam.

Maillard, S., Gottstein, B., Haag, K., Ma, S., Colovic, I., Benchikh-Elfegoun, M. C., Knapp, J., and Piarroux, R. (2009). The tandemly repeated multilocus microsatellite EmsB : a new tool to investigate the genetic diversity of Echinococcus granulosus sensu lato. Fournal of Clinical Microbiology. Epub ahead of print.
Monis, P. T., Andrews, R. H. and Saint, C. P. (2002). Molecular biology techniques in parasite ecology. International Fournal for Parasitology 32, 551-562.

Nakao, M., McManus, D. P., Schantz, P. M., Craig, P. S. and Ito, A. (2007). A molecular phylogeny of the genus Echinococcus inferred from complete mitochondrial genomes. Parasitology 134, 713-722.

Nakao, M., Sako, Y. and Ito, A. (2003). Isolation of polymorphic microsatellite loci from the tapeworm Echinococcus multilocularis. Infection, Genetics and Evolution 3, 159-163.

Okamoto, M., Oku, Y., Kurosawa, T. and Kamiya, M. (2007). Genetic uniformity of Echinococcus multilocularis collected from different intermediate host species in Hokkaido, Japan. Fournal of Veterinary Medical Science 69, 159-163.

Posselt, A. (1900). Die geographische Verbreitung des Blasenwurmleidens insbesondere des Alveolarechinokokkus des Leber und dessen Casuistik seit 1886. Enke, Stuttgart.

Ricklefs, R. E. and Miller, G. L. (2000). In Ecology W. H. Freeman, New York.

Rinder, H., Rausch, R. L., Takahashi, K., Kopp, H., Thomschke, A. and Loscher, T. (1997). Limited range of genetic variation in Echinococcus multilocularis. Fournal of Parasitology 83, 1045-1050.

Roratto, P. A., Bartholomei-Santos, M. L., Gutierrez, A. M., Kamenetzky, L., Rosenzvit, M. C., and Zaha, A. (2006). Detection of genetic polymorphism among and within Echinococcus granulosus strains by heteroduplex analysis of a microsatellite from the U1 snRNA genes. Genetics and Molecular Research 5, 542-552.

Saarma, U., Jogisalu, I., Moks, E., Varcasia, A., Lavikainen, A., Oksanen, A., Simsek, S., Andresiuk, V., Denegri, G., Gonzalez, L. M., Ferrer, E., Garate, T., Rinaldi, L. and Maravilla, P. (2009). A novel phylogeny for the genus Echinococcus, based on nuclear data, challenges relationships based on mitochondrial evidence. Parasitology 136, 317-328.

Scott, J. C., Stefaniak, J., Pawlowski, Z. S. and McManus, D. P. (1997). Molecular genetic analysis of human cystic hydatid cases from Poland: identification of a new genotypic group (G9) of Echinococcus granulosus. Parasitology 114, 37-43.

Smith, J. E. (2009). Chapter 6. Tracking Transmission of the Zoonosis Toxoplasma gondii. Advances in Parasitology 68, 139-159.

Takumi, K., de Vries, A., Chu, M. L., Mulder, J., Teunis, P. and van der Giessen, J. (2008). Evidence for an increasing presence of Echinococcus multilocularis in foxes in The Netherlands. International Fournal for Parasitology 38, 571-578.

Templeton, A. R. (2008). The reality and importance of founder speciation in evolution. Bioessays 30, 470-479.

Thompson, R. C. and McManus, D. P. (2002). Aetiology: parasites and live-cycles. In: WHO/OIE Manuel on echinococcosis in humans and animals : a public health problem of global concern (ed. Eckert, J., Gemmell, M. A., Meslin, F. X. and Pawlowski, Z. S.), pp. 1-19. World Organization for Animal Health, Paris, France. 
van Herwerden, L., Gasser, R. B. and Blair, D. (2000). ITS-1 ribosomal DNA sequence variants are maintained in different species and strains of Echinococcus. International Fournal for Parasitology 30, 157-169.

Vuitton, D. A., Zhou, H., Bresson-Hadni, S., Wang, Q., Piarroux, M., Raoul, F. and Giraudoux, P. (2003).
Epidemiology of alveolar echinococcosis with particular reference to China and Europe. Parasitology 127 (Suppl), S87-S107.

Yang, Y. R., Rosenzvit, M. C., Zhang, L. H., Zhang, J. Z. and McManus, D. P. (2005). Molecular study of Echinococcus in west-central China. Parasitology 131, 547-555. 